\title{
Semi-empiric noise modeling of a Cargo eVTOL UAV by means of system identification from flight noise measurement data
}

\author{
Michael Schmähl $^{1}\left[\right.$ Christian Rieger $^{1} \cdot$ Sebastian Speck $^{1} \cdot$ Mirko Hornung $^{1}$
}

Received: 22 March 2021 / Revised: 27 August 2021 / Accepted: 22 September 2021 / Published online: 2 December 2021

(c) The Author(s) 2021

\begin{abstract}
This publication shows the semi-empiric noise modeling of an electric-powered vertical takeoff and landing (eVTOL) unmanned aerial vehicle (UAV) by means of system identification from flight noise measurement data. This work aims to provide noise models with a compact analytical ansatz for horizontal and vertical flight which are suited for integration into a geographical information system. Therefore, flight noise measurement campaigns were conducted and evaluated. An existing noise model ansatz is adapted to the eVTOL UAV under consideration and noise models are computed from the measurement data using the output error method. The resulting models are checked for plausibility by comparing them to technical literature. The horizontal flight noise model is subjected to a correlation analysis and the influence of meteorological effects are examined. To achieve a higher level of accuracy in future noise modelings, an optimization of the microphone positions as well as the flight trajectory is carried out.
\end{abstract}

Keywords Noise modeling $\cdot$ Flight noise measurement $\cdot$ UAV $\cdot$ eVTOL $\cdot$ Urban air mobility $\cdot$ Output error method

\section{Introduction}

The use of aerial vehicles necessarily leads to a certain amount of noise emissions. Noise models are required to quantify this noise impact and therefore they are a precondition to consider noise in planning processes. Urban air mobility (UAM) challenges political decision-makers and public authorities, as it will, in addition to road traffic, lead to further traffic noise emissions in the urban area. For this reason, flight noise models are required to represent the noise impact of UAM aircraft movements.

For conventional air traffic noise in the vicinity around airports, multiple software tools exist. The AEDT is a wellknown example which is qualified for legislation processes with the focus on noise-protection zones, land-use planning, and consulting [1]. Cargo eVTOL (electric powered vertical takeoff and landing) UAVs (unmanned aerial vehicle) operate in much closer distances to urban areas. Established tools that are suitable for computing noise contours of such aircraft on geodata of urban areas do not yet exist, but due

Michael Schmähl

michael.schmaehl@tum.de

1 Technical University of Munich, 85748 Garching, Germany to the progress of UAM applications there is a need for such tools in the near future.

A possible approach to meet this need is to integrate UAV noise models into existing geo information system (GIS) software. As existing GIS software is not primarily suited for noise computations, the complexity of the noise models should be kept as low as possible. In many cases cargo eVTOL UAVs operate at flight Mach numbers smaller than 0.1 and their range of audibility is significantly smaller than $1 \mathrm{~km}$ and, thus, the Doppler effect, meteorological effects on sound propagation and sound wave run times are negligible.

Traditional rotorcraft noise models might generally be eligible for reproducing eVTOL UAV noise. A prominent example of such a tool is NASA's Rotorcraft Noise Model (RNM) [2] which is based on sound hemispheres that can, e.g., be based on ground noise measurement data. Meteorological effects on the sound propagation are considered in this approach which simulates the sound propagation from the source to terrain data in a time-based manner. Another example is FRAME [3] which is a parametric semi-empirical helicopter noise model capable of resolving transient maneuver noise. The noise model identification in this approach is based on de-Dopplerized noise data, and propagation effects are neglected. A simple but yet robust semiempiric method for noise footprint modeling of UAV s can 
be found in [4]. Like in FRAME the model identification is based on de-Dopplerized noise data and meteorological effects on sound propagation are neglected. Additionally, this approach is neglecting sound wave run times and, thus, can be characterized as an emission noise model. Due to the fact that this model is parametric is adjustable to arbitrary UAVs if noise data for model identification exists.

This paper presents an adaption and extension of the noise modeling approach first published in [4] to an eVTOL UAV which considers vertical and horizontal flight phases. The model aims to predict the A-weighted instantaneous over all sound pressure level (OASPL). Due to the simplifications this approach involves, it is well suited for integration into a GIS software.

The paper starts with a description of the mathematical modeling approach, explaining the basic model ansatz and the mathematical background of the system identification procedure. Furthermore, the acquisition and processing of noise and flight measurement data are described. In a next step, the adaption of the model to the eVTOL UAV under consideration in this work and the corresponding noise model results is presented. An error analysis is conducted and based on a correlation analysis of the synchronized noise and flight measurement data, an optimization of the microphone positions and the flight trajectory is presented.

\section{Modeling approach}

The intended application of the noise model demands a compact analytical modeling approach. The UAV states need to be considered as parameters in this approach. This ensures that noise emissions are predictable in each flight state. For these reasons a semi-empiric noise model, which is determined from flight noise measurement data by means of system identification, is well suited, as suggested in [4]. The mentioned approach is a point source emission model which is capable of predicting the maneuver-specific emitted noise.

\subsection{Semi-empiric noise model by means of system identification of flight noise measurement data}

The modeling approach according to [4] consists of an analytical equation which has the structure of the formula for sound pressure level (SPL) addition:

$L_{i}(d, \bar{\varphi}, \vartheta, \vec{z}, \vec{p})=10 \cdot \log _{10}\left(10^{\left(l_{\text {directivity }}+l_{\text {thrust }}+l_{\text {spreading }}\right)^{1 / 10}}+10^{\left(L_{\text {background }}\right)^{1 / 10}}\right)$

The result of (1) corresponds to the sound pressure level $L_{i}$ at observer position $i$ which is emitted depending on the distance between observer and $\operatorname{UAV} d$, azimuth $\varphi$ and polar angle $\vartheta$ of the UAV noise emission, the UAV states $\vec{z}$ and the free parameter vector $\vec{p}$ (Fig. 1). The following holds for the different sound pressure level contributions:

$l_{\text {directivity }}=f(\bar{\varphi}, \vartheta, \vec{p})$

$l_{\text {thrust }}=f(\vec{z}, \vec{p})$

$l_{\text {spreading }}=f(d, \vec{p})$

$L_{\text {background }}=f(\vec{p})$

The sound pressure level of the UAV $L_{\mathrm{UAV}}$ consists of three contributions:

$L_{\mathrm{UAV}}=l_{\text {directivity }}+l_{\text {thrust }}+l_{\text {spreading }}$

The model approach combines formula describing the physical relationships of sound generation and sound propagation with UAV states and free parameters which are to be determined by system identification. Thus, the modeling approach is a gray box approach.

Furthermore, [4] suggests computing the parameters with the output error method (OEM) making use of a maximum likelihood estimator (MLE) which shows high robustness against normally distributed measurement noise.

To preserve compactness and efficiency of the model, its noise predictions underlie the following restrictions:

- Meteorological influence on sound propagation is neglected.

- Free field noise is predicted, and sound reflection and scattering are neglected,

- The Doppler effect on sound propagation is neglected.

- The run time difference of sound signals between UAV and observer is neglected.

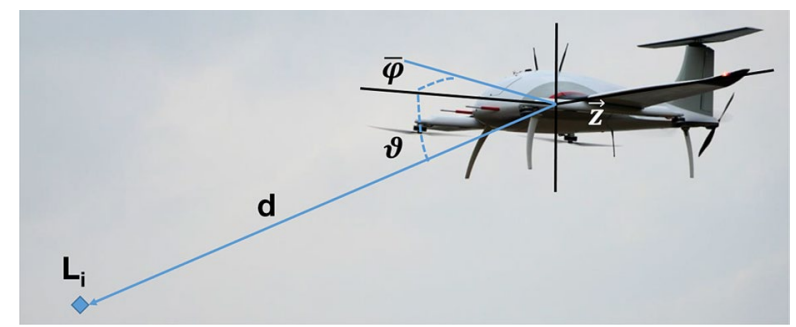

Fig. 1 Schematic representation, picture taken from [15] and edited 


\subsection{Mathematic foundations of OEM and MLE}

The foundations of OEM and MLE can be found in [6]. The OEM determines the parameter vector $\vec{p}$ such that the residuum between noise measurement data at microphone position $i$ and noise model prediction $L_{i}$ tends to a minimum. For this reason, it is also called 'response curve fitting'.

The MLE estimates the parameter vector in such a way that a vanishing noise model deviation

$L_{i, \text { model }}(\vec{p}, t)-L_{i, \text { measurement data }}(t)=0$

shows the highest probability for all points of time $t$. The MLE possesses several desirable properties of an estimator. It is asymptotically unbiased, consistent, normally distributed and efficient. In case of statistically independent flight noise measurement data, it can be expected that, with increasing number of measurement data points, the estimation of $\vec{p}$ converges to the true value [6, p. 83]. As a precondition the measurement noise is required to be statistically independent as well [6, p. 84]. Furthermore, the input data, which is $d, \bar{\varphi}, \vartheta$ and $\vec{z}$ in the case of a noise model, needs to be sufficiently and adequately distributed and statistically independent to excite the full spectrum of system behavior regarding noise emission [6, p. 83f.].

In this context, two requirements for a model postulate can be deduced: ideally, it needs enough degrees of freedom to be able to reproduce all physical effects that the measurement data contains to avoid process noise [6, p. 124].

Furthermore, the parameters are to be chosen in such a way that they are linearly independent and that they sufficiently determine the model [6, p. 377]. The statistic accuracy of how well the model matches the measurement data is determined by the parameter error covariance matrix [6, p. 111]. The matrices' diagonal elements are a measure of the parameter estimation's accuracy, and its other elements are a measure of the degree of linear dependency among the parameter estimates.

\section{Noise data acquisition}

Obtaining reliable MLE estimations presumes high-quality data acquisition. During project "Demueb Phase 3", to meet these requirements, a noise measurement system was deployed at the IAD. The three measuring stations recorded the noise immissions with capacitive microphones of type 'Microtech Gefell MK221/MV203' and digitalized it in a sound card. In parallel GPS receivers measured the microphone positions and recorded the GPS-exact time. Hereby, an exact spatial and time-wise characterization of the noise immissions was made possible. The measurement microphone was directly located above a steel measurement plate to exclude the influence of uncontrolled reflections (Fig. 2). Free field sound was calculated according to [7]. The measurement microphone was calibrated with a class 1 calibrator before the measurements. To exclude the influence of the measuring system temperature on the data, all components of the system were sufficiently warmed up. Regular recalibrations between the measurements did not manifest significant deviations.

Within this work, noise data of the cargo eVTOL UAV "Manta Ray" by Phoenix-Wings was acquired. This aircraft had an average cruise speed of $23.6 \mathrm{~m} / \mathrm{s}$ during the measurement campaign. It has a wing span of $3.53 \mathrm{~m}$ and a maximum takeoff mass of $35 \mathrm{~kg}$ [8].

The positioning of the measuring stations was improved manually during first measurement flights to acquire as many relevant flight states as possible.

Additionally, one of the measuring stations recorded air temperature, humidity as well as wind direction and wind speed.

Within this work several measurement campaigns were conducted together with a project partner during which two eVTOL UAVs in different sizes, configurations and modifications were investigated. Figure 3 shows the Manta Ray UAV. Its noise model is the subject of this publication.

While horizontally flying by the measuring stations, acoustic data of various emission angles of the eVTOL UAV are recorded. For an increased number of measuring points, the fly-bys were conducted for various distances, flight altitudes and flight directions. During these flights, speed was varied in the range of $22-30 \mathrm{~m} / \mathrm{s}$ resulting in a nondimensional power consumption range of 0.9-1.4.

During hover flight, the same was achieved through hovering at various positions. Yawing slowly at one position did allow to acquire the noise emissions for all azimuth angles at this position. In case the position is held during flight, this is referred to as hover flight in the following. In case of climbing and sinking, it is referred to as vertical flight.

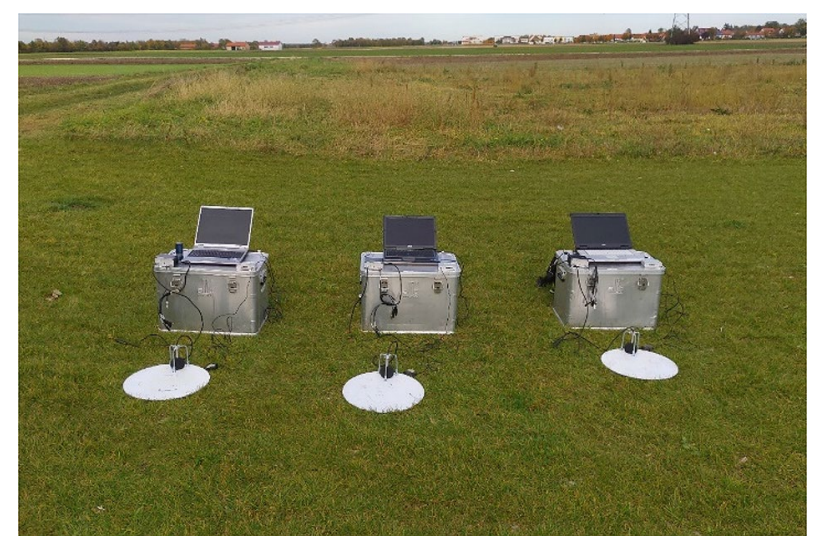

Fig. 2 All three measuring stations (meteorology measuring equipment not displayed) 


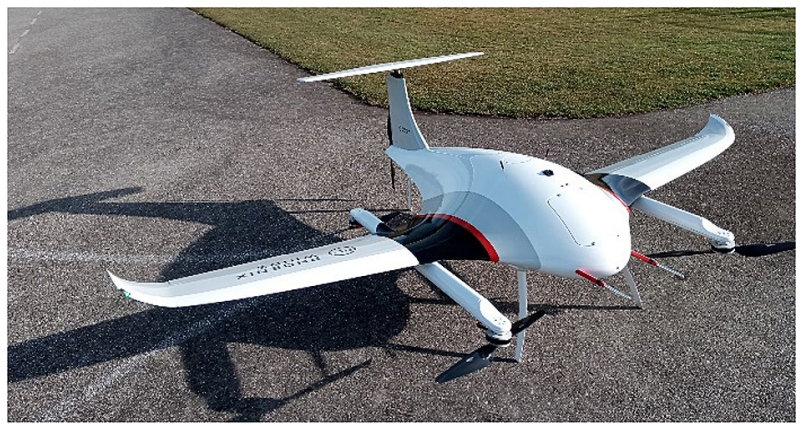

Fig. 3 Phoenix-wings eVTOL UAV, Manta Ray”

The pre-processing of the noise measurement data for the noise model calculation through system identification comprises the following steps: synchronization with flight data, third octave band decomposition, A-weighting of the noise data, computation of A-weighted overall sound pressure level as well as translation of the time vector from microphone immission time to UAV emission time. All in all, two complete Manta Ray flights were used for the noise modeling work. Figure 4 displays the waterfall diagram of the second flight. During horizontal flight, the blade passing frequency and its parallelly shifted harmonics can be recognized as 'parallel lines' upward of $160 \mathrm{~Hz}$. The nature of the noise is predominantly tonal. While vertically climbing and sinking, these 'tonal lines' are still present while the noise exhibits an all in all stronger broadband nature. In the middle of the flight, a jet aircraft takeoff took place at a nearby airport, and this event is clearly visible in the waterfall diagram.

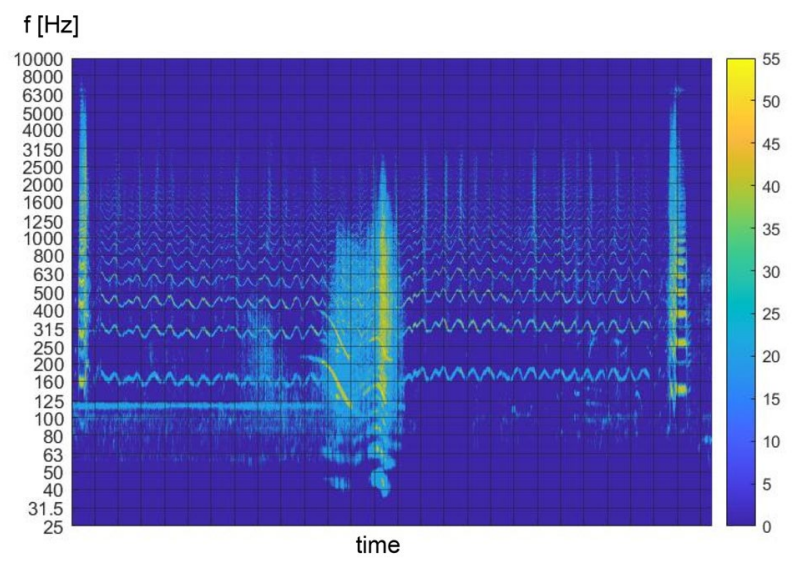

Fig. 4 Waterfall diagram [SPL in $\mathrm{dB}(\mathrm{A})$ ] of one complete flight lasting $14 \mathrm{~min}$

\section{Modeling}

\subsection{Model approach determination}

The first step of the model determination is to adjust Eq. (1) to the UAV under consideration. $l_{\text {thrust }}$ is based on the logarithmic correlation between shaft power and sound pressure level $\left[9\right.$, p. 32], [4, p. 3]. $l_{\text {directivity }}$ is mainly influenced by installation effects. This term was formulated as a polynomial that models the characteristic of the UAV noise directivity with a minimum number of parameters (Eq. 3). This directivity is characterized by a sound pressure level which increases for decreasing polar angles and by the existence of a sound pressure level maximum for one distinct azimuth value, see (Fig. 5).

Equation (1) was adjusted to give it a more favorable shape with regard to OEM mathematics:

$L_{i}(d, \bar{\varphi}, \vartheta, \vec{z}, \vec{p})=10 \cdot \log _{10}\left(\begin{array}{l}10^{\left(l_{\text {directivitythrust }}+l_{\text {spreading }}\right)} \\ +10^{\left(L_{\text {background }}\right)} 1^{1 / 10}\end{array}\right)$

Merging the directivity and the thrust term avoids that one of them is set to zero by the OEM. The single model approach terms are formulated as follows:

$$
\begin{gathered}
l_{\text {directivity+thrust }}\left(\bar{\varphi}, \vartheta, P_{e l}, \vec{p}\right)=p_{1} \cdot \log \left(P_{e l}\right) \cdot\left(1+p_{2}\right. \\
\left.\cdot \sin \left(\bar{\varphi}-p_{3}\right) \cdot\left(\vartheta+\frac{\pi}{2}\right)\right) \cdot\left(1+p_{4} \cdot \sin \left(\vartheta-p_{5}\right)\right)
\end{gathered}
$$

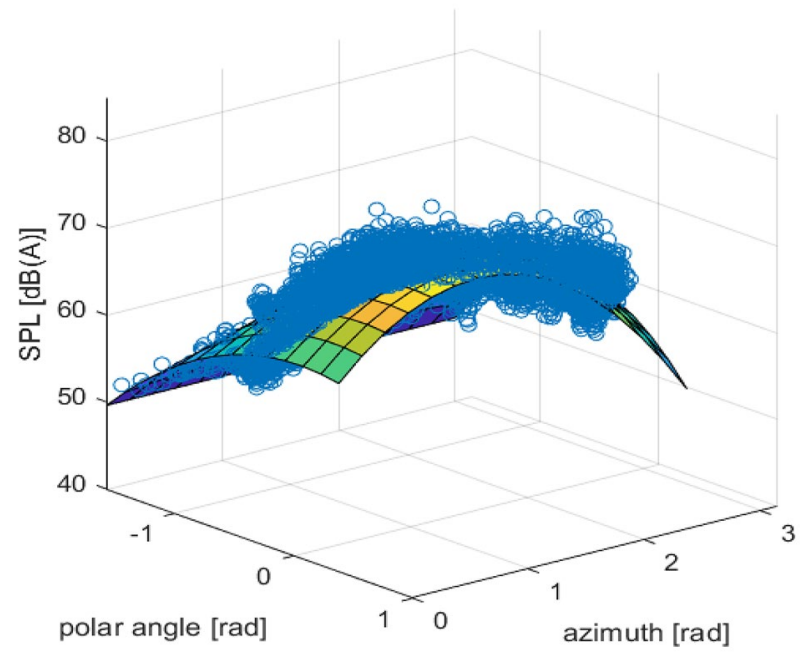

Fig. 5 Blue points: all horizontal flight noise measurement points corrected to a reference distance of $30 \mathrm{~m}$; surface: best fit polynomial surface of fifth order 
$l_{\text {spreading }}(d, \vec{p})=-p_{6} \cdot 20 \cdot \log \left(\frac{\left|\overrightarrow{r_{i}^{N E D}}\right|}{40 \mathrm{~m}}\right)$

$L_{\text {background }}(\vec{p})=p_{7} / d B$

$\vec{z}$ reduces to the scalar quantity $P_{e l}$. This quantity corresponds to the nondimensional electric power consumption of the UAV which is dominated by the power consumption of the propeller. A parameter error covariance analysis did reveal that a further increase in the number of parameters in term $l_{\text {directivity }+ \text { thrust }}$ leads to a higher level of linear dependence among the parameters. This is undesirable with regards to the model quality.

\subsection{Result analysis}

The model approach was applied to the horizontal flight as well as to the climbing and the sinking vertical flight. During large parts of the flight time horizontal flight is taking place, thus, a sufficient database for OEM based parameter estimation exists for this flight phase. The vertical flight models are mainly based on the vertical takeoffs and landings that were flown by the autopilot at the beginning and the end of each flight. As this data does not sufficiently cover the emission angle space, further measurement data segments of manually flown vertical flights had to be included. During these manual flights high propeller speed variations occurred. It happened frequently that the pilot had to counter steer wind gusts in order to hold the position of the UAV during hover flight which lead variations in the propeller speed. Because of the contamination with these variations, the manual measurement data was to large parts not usable for the modeling process.

The parameter values of the three flight noise models are listed in the table below. The magnitude of the background noise is concordantly estimated to a value of $40 \mathrm{~dB}$ (A). Thus, this value is consistent and agrees with the observation made in the noise measurement data.

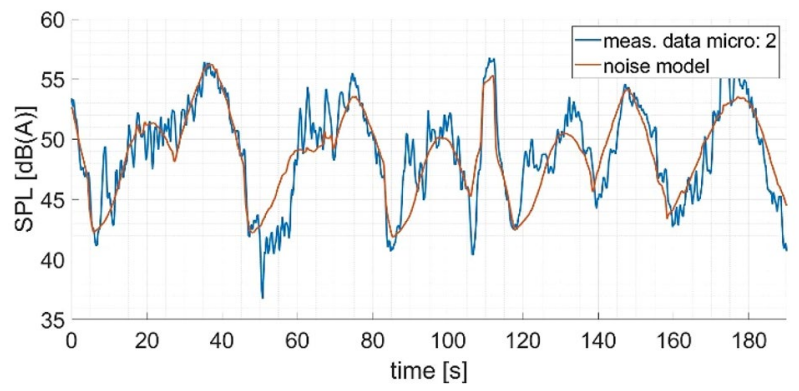

Fig. 6 Noise measurement data vs. noise model (horizontal flight model)
Table 1 Parameter values of noise models

\begin{tabular}{lrrr}
\hline Parameter & Horizontal flight & $\begin{array}{l}\text { Vertical flight } \\
\text { (climb) }\end{array}$ & $\begin{array}{l}\text { Verti- } \\
\text { cal flight } \\
\text { (sink) }\end{array}$ \\
\hline $\mathrm{p}_{1}$ & 13.409 & 16.726 & 12.722 \\
$\mathrm{p}_{2}$ & 0.176 & 0.090 & 0.146 \\
$\mathrm{p}_{3}$ & -0.280 & 0.137 & 0.085 \\
$\mathrm{p}_{4}$ & -0.365 & -0.151 & -0.513 \\
$\mathrm{p}_{5}$ & 1.049 & 0.293 & 0.411 \\
$\mathrm{p}_{6}$ & 0.939 & 1.000 & 0.800 \\
$\mathrm{p}_{7}$ & 40.393 & 40.162 & 40.278 \\
\hline
\end{tabular}

Figure 6 shows that the horizontal flight model well resolves the trend of the noise measurement data. Model deviations occur regularly and reach orders of magnitude of circa $5 \mathrm{~dB}$ which is to be expected as the measured SPLs are relatively close to the SPL of the background noise (see $\mathrm{p}_{7}$ in Table 1).

Figure 7 shows a two-dimensional representation of the sound pressure level which covers the entire noise emission angle space. Thereby, it represents the directivity of the UAV noise emission.

Comparison with Fig. 5 points out that this directivity is in good agreement with that which can be observed in the noise measurement data. According to theory the sound pressure level that a propeller emits in an undisturbed air stream is negligible in axis directions and reaches its maximum under an angle of $120^{\circ}$ relative to the propeller axis [9, p. 6] and it is axially symmetric.

The value of $p_{3}$ has a negative sign. Inserting this value in formula (3) exhibits that the sound emission reaches its maximum under an angle of $74^{\circ}$ relative to the propeller axis. This finding contradicts the theory of a propeller in an undisturbed air stream, but agrees with [10]. The mentioned source shows a pusher propeller whose inflow is disturbed by a pylon. Only from emission angles smaller than $90^{\circ}$ relative to the propeller axis on there is a significant sound pressure level increase compared to the undisturbed inflow at medium thrust. This observation confirms the plausibility of the horizontal flight noise model and suggests that interaction noise from the disturbed propeller inflow is causal for the sound pressure level maxima which point by $16^{\circ}$ into the flight direction.

From 0 down to $-\pi / 2$, decreasing polar angle values lead to decreasing sound pressure values which also contradicts the theory of a rotationally symmetric propeller noise emission. However, an aeroacoustic study of a pusher propeller configuration in [11] demonstrates how a disturbed propeller inflow, as it is observed for the Manta Ray UAV, leads to a significant loss of axis symmetry in propeller noise emission. 


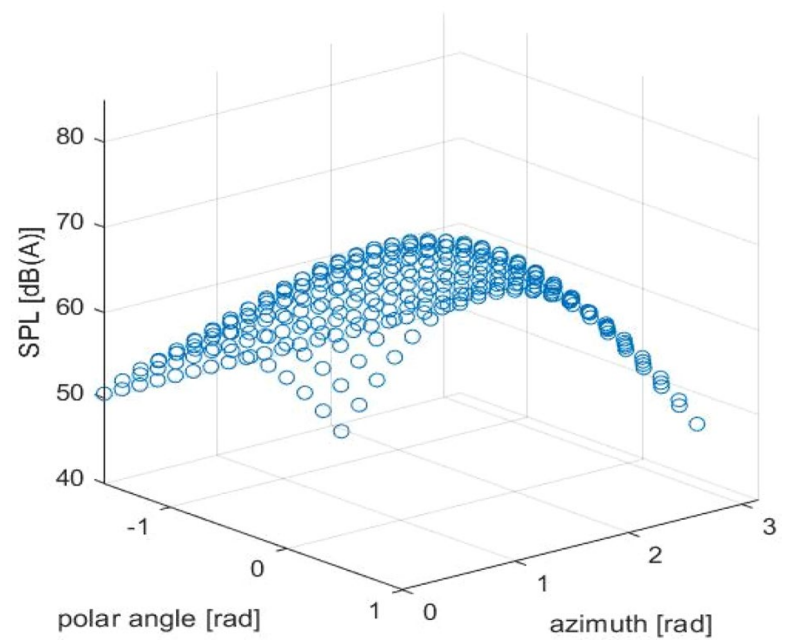

Fig. 7 Sound pressure level at a reference distance of $30 \mathrm{~m}$ and a nondimensionalized electric power consumption of 1.1 (horizontal flight model)

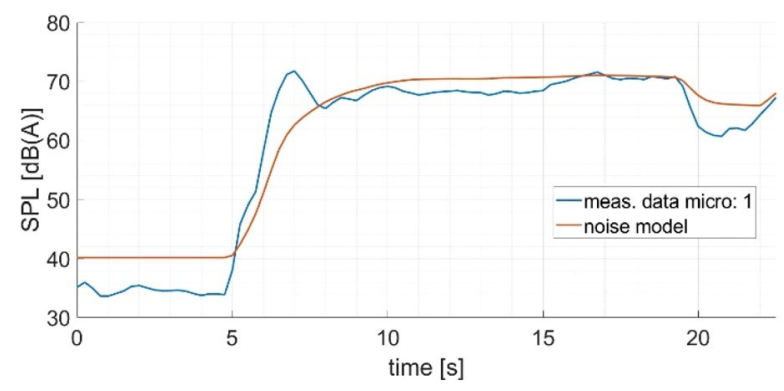

Fig. 8 Noise measurement data vs. noise model (climbing vertical flight model)

Compared to the horizontal flight models, the vertical flight models reproduce the trend of the noise measurement data less accurately. Figure 8 shows a vertical takeoff phase until that point of time when the pusher propeller fan speed is not negligible anymore. This marks the beginning of the transition. Long lasting deviations of 3-10 dB occur and the sound maximum at $7 \mathrm{~s}$ is not reproduced by the model.

From Fig. 9 is can be seen that the climbing vertical flight model has no distinct directionality. The before mentioned strongly directional theoretic propeller noise emission is valid for conventional propellers with moderate blade tip Mach numbers and flight speeds [9, p. 6]. Under these operating conditions, mainly tonal noise is generated. In vertical and hover flight this model is less valid, as effects like blade vortex interaction appear in a more turbulent flow regime that leads to additional noise which has a rather broadband nature [12].

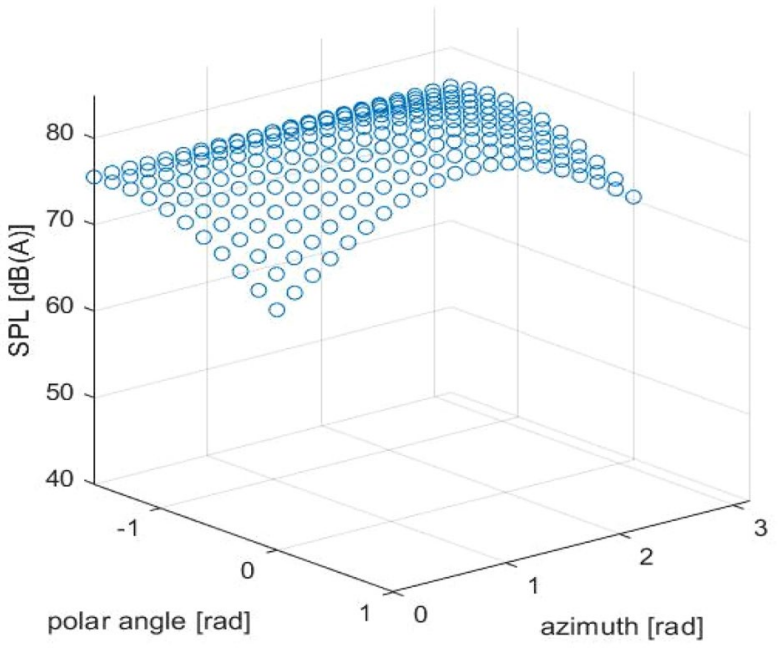

Fig. 9 Sound pressure level at a reference distance of $30 \mathrm{~m}$ and a nondimensionalized electric power consumption of 6.5 (climbing vertical flight model))

\section{Error analysis}

\subsection{Potential sources of error}

High deviations of noise models can be caused by the noise measurement data. If measurement noise, disturbances or the measurement data are not statistically independent, the properties of an estimator, as summarized in chapter 2.2, do not hold. In this case an estimation cannot be assumed to be bias free, a wrong estimate is the consequence. The same is true for a sufficient and adequate variation of the control inputs in the measurement data. In the sense of a noise model distance $d$, azimuth $\bar{\varphi}$ and polar angle $\vartheta$ of the UAV noise emission and $P_{e l}$ are referred to as control inputs. Within the modeling process only noise measurement data sequences that are free from disturbing noise events were used.

Further reasons for high deviations can be traced back to the model approach and its parametrization. If the model approach fails to resolve physical effects which are present in the measurement data then this will lead to process noise. An over- or underdetermined parametrization can be a reason for that as well.

\subsection{Vertical flight models}

Process noise is the main cause for the high deviations of the vertical flight models. The complexity of noise emission in vertical and hover flight is high due to the high propeller speed variations and due to the fact that four propellers contribute to the emissions. Phase offsets of the propellers can, e.g., lead to interference patterns; thus, a model approach which does not account for propeller speed and 


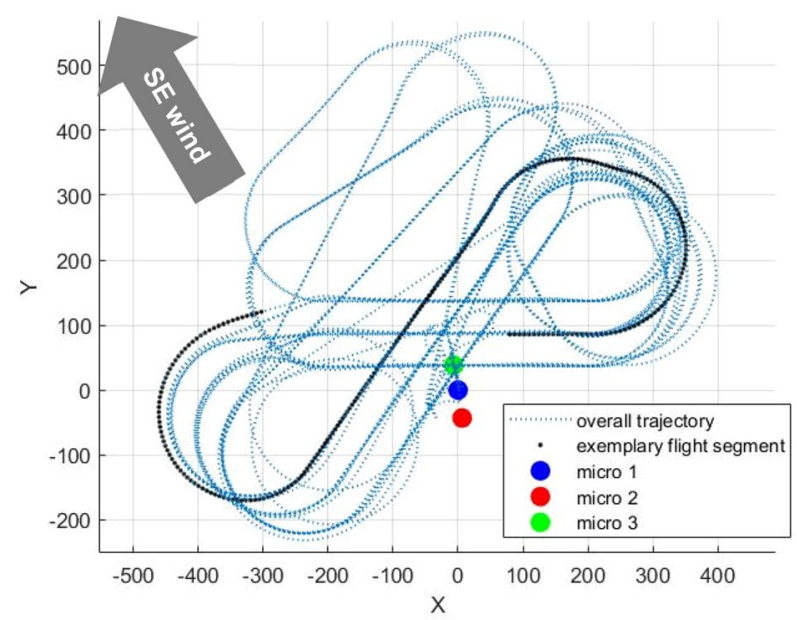

Fig. 10 Overall flight trajectory (blue), examined segment (black) and microphone positions (in ENU coordinates)

phase-dependent noise interference patterns necessarily leads to process noise. The method of iterative model approach postulation, model parameter determination via OEM and assessment of the model quality did not lead to any accuracy increase in the vertical flight models anymore. For this reason, a fundamental investigation is necessary to clarify the effects that dominate the sound emission in vertical and hover flight of eVTOL UAVs. Requirements for a future model approach are to be derived from these findings.

\subsection{Horizontal flight model}

The horizontal flight model is in much better agreement with the noise measurement data than the vertical flight models. The existing deviations were analyzed and presented in the following.

\subsubsection{Analysis of an exemplary flight segment}

The trajectory of the horizontal flight is a recurring loop pattern. To identify flight segments with significant noise model deviations, one such segment is examined in the following (Fig. 10). The measured wind speed averages $13.0 \mathrm{~km} / \mathrm{h}$ and the nondimensional electric power consumption is above 1.3 during large parts of the flight. At the beginning of the segment, the UAV flies in the easterly direction away from the microphones. The course of the bank angle curve (Fig. 11) shows that the UAV is in turning flight during $t=5-27 \mathrm{~s}$ und $t=46-63 \mathrm{~s}$. The sound pressure level curve (Fig. 12) exhibits straight flight noise model residuals being significantly smaller than the turning flight noise model residuals, e.g., during the second turn a deviation of more than $5 \mathrm{~dB}$ occurs which is more than $8 \mathrm{~s}$ long. From the offset trajectory at decreasing electric power consumption (Fig. 13) and

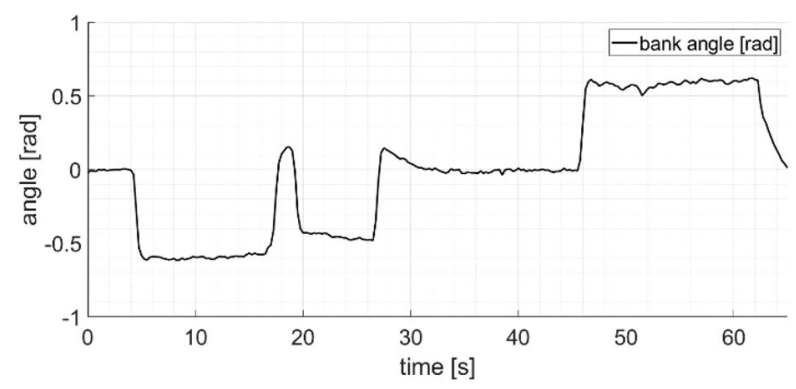

Fig. 11 Bank angle

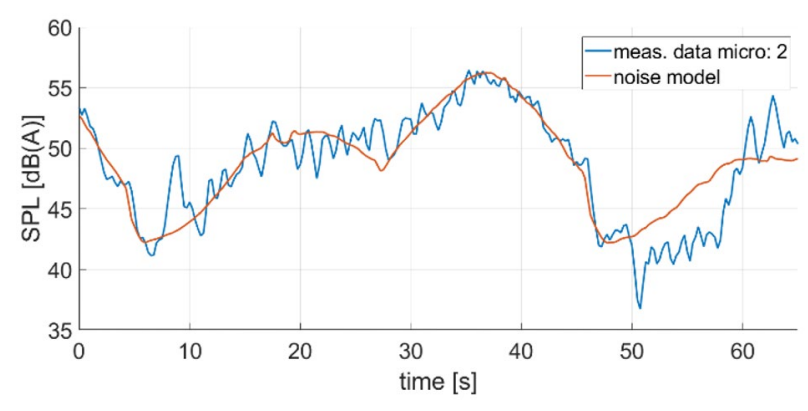

Fig. 12 SPL curve

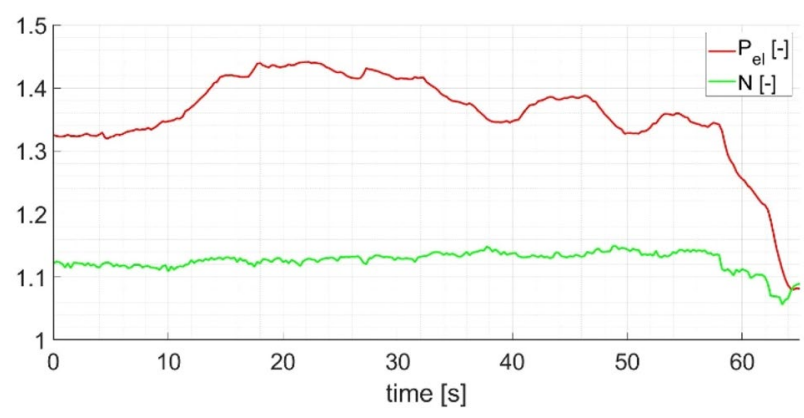

Fig. 13 Electric power consumption and propeller speed (nondimensional)

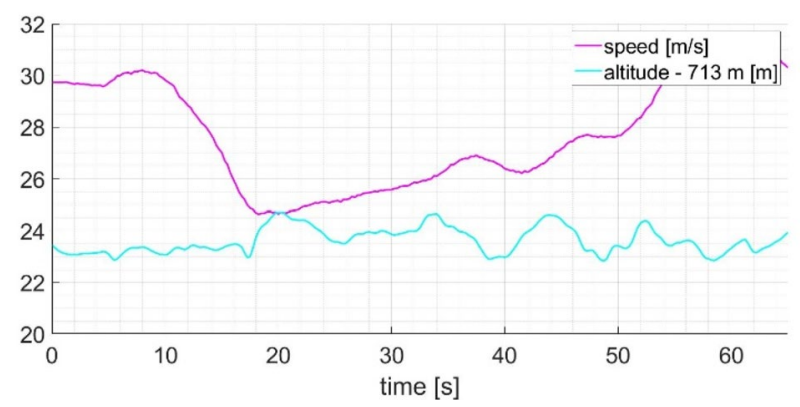

Fig. 14 Ground speed and altitude 
still increasing velocity (Fig. 14), it can be seen that the UAV was driven away by wind in this second turn. A comparison of the noise model deviations with an identical trajectory segment at a wind speed of $7.6 \mathrm{~km} / \mathrm{h}$ shows no wind drift but, the noise model residuals increase during turning flight in a similar way. Consequently, wind is not the cause for the noise model residuals in turning flight in (Fig. 12). All in all, the noise model follows the measurement data trend in turning flight as well, but in comparison to straight flight it cannot follow the higher sound pressure level fluctuations.

During turning flight, a non-axial propeller inflow which also deviates from the straight flight situation due to rudder deflections is to be assumed. In combination with the higher sound pressure level fluctuations, these findings plead for increased interaction noise during turning flight. As the noise model approach does not account for this additional kind of noise, the resulting noise model deviations are to be interpreted as process noise in the sense of system identification.

\subsubsection{Correlation analysis}

In "Analysis of an exemplary flight segment" turns are identified as flight segments with increased noise model deviations; hence, the question is in how far there are systematic relationships between noise measurement data, noise model deviations, UAV-states, distance and noise emission angles.

A correlation analysis of the measurement data underlying the noise modeling is conducted to clarify this question. The vector of the statistic variable $\vec{X}$ consists of eight components: UAV speed, propeller speed, electric power consumption, band angle, distance UAV to microphone, polar angle and azimuth of noise emission and noise model deviation. The correlation matrix

$\operatorname{Corr}(\vec{X})=\frac{\operatorname{Cov}\left(X_{i}, X_{j}\right)}{\sqrt{\operatorname{Var}\left(X_{i}\right) \cdot \operatorname{Var}\left(X_{j}\right)}} s$

with sample covariance

$\operatorname{Cov}\left(X_{i}, X_{j}\right)=\frac{1}{N_{t}} \cdot \sum_{t=1}^{N_{t}}\left(X_{i}(t)-\overline{X_{i}}\right) \cdot\left(X_{j}(t)-\overline{X_{j}}\right)$

and sample variance

$\operatorname{Var}\left(X_{i}\right)=\frac{1}{N_{t}} \cdot \sum_{t=1}^{N_{t}}\left(X_{i}(t)-\bar{X}_{i}\right)^{2}$

calculates from the number of discrete data samples $N_{t}$. The nondiagonal entries $\operatorname{Corr}\left(X_{i}, X_{j}\right)$ with $i \neq j$ exhibit values between -1 and 1 . At a value of -1 and 1 a negative, respectively, positive linear relationship between the statistic variables under consideration exists and at a value of 0 there is no relationship. The result of the analysis is displayed in Fig. 15. The correlation matrix shows the obvious relationship between UAV speed, propeller speed and electric power consumption.

Furthermore, a strong correlation between bank angle, distance and polar angle of sound emission exists which can be explained by the fact that the microphones are located in the middle of the overall trajectory. During turning flight, there is in most cases an average distance of $400 \mathrm{~m}$ between the UAV and the microphones; thus, the bank angle is coupled to the polar angle of noise emission in these cases. In agreement with Sect. 5.3.1 these three quantities are weakly correlated with the noise model deviation and thereby statistically prove that it increases in turning flight.

Both, distance and polar angle of noise emission are considered in the noise model and consequently the flight noise measurement data is not statistically independent. As this is a precondition of the MLE, a loss of accuracy in the noise modeling is to be expected.

The statistical dependency of the measurement data reduces the number of linearly independent parameters which the MLE can determine in a noise model. This limitation of the model approach in its ability to reproduce the actual system behavior necessarily promotes process noise.

\subsubsection{Atmospheric damping}

Meteorological factors can influence the noise measurement data. To keep the influence of wind at a minimum, measurements were only conducted on low-wind days. Maximum wind speeds of $10 \mathrm{~km} / \mathrm{h}$ were targeted.

When sound waves propagate in air, molecular absorption leads to a sound pressure level decrease that depends on run length, frequency and humidity. This effect is referred to as atmospheric damping in the following. The frequency dependent damping values are calculated according [13, p. 197f] for the meteorological conditions of the second flight (Fig. 16). For the blade passing frequency of $160 \mathrm{~Hz}$ and its first harmonic, the influence of atmospheric damping is significantly lower than $1 \mathrm{~dB}$ at distances of $400 \mathrm{~m}$. In contrast to that, atmospheric damping gains importance for higher harmonics and broadband noise. The noise measurement data of the second flight is corrected with the curve displayed in Fig. 17 and a noise model is determined. Compared to the noise model based on not corrected noise data, no significant change in the model prediction is observed. In the time interval from 50 to $60 \mathrm{~s}$ the model deviation decreases to values of $1-2 \mathrm{~dB}$. To exclude possible process noise through atmospheric damping in future modelings, a noise measurement correction should be considered, especially if run lengths of several hundreds of meters occur. 


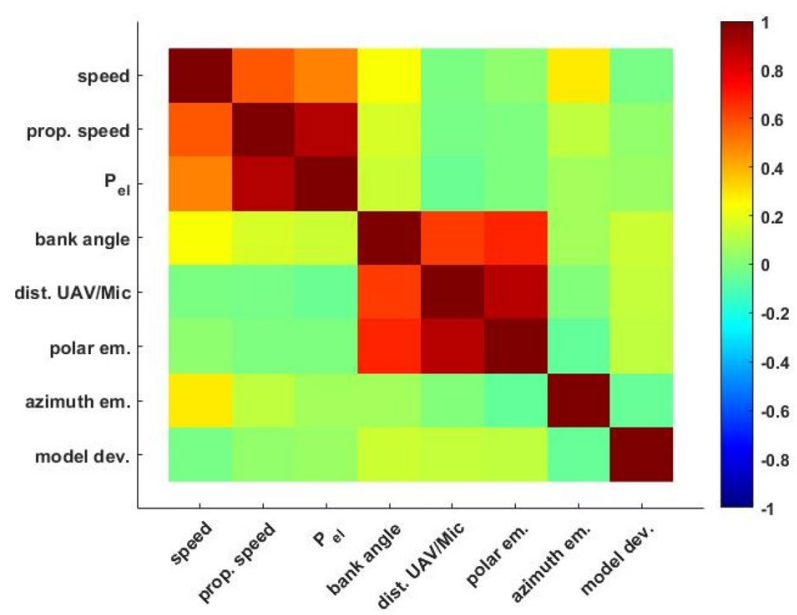

Fig. 15 Correlation matrix

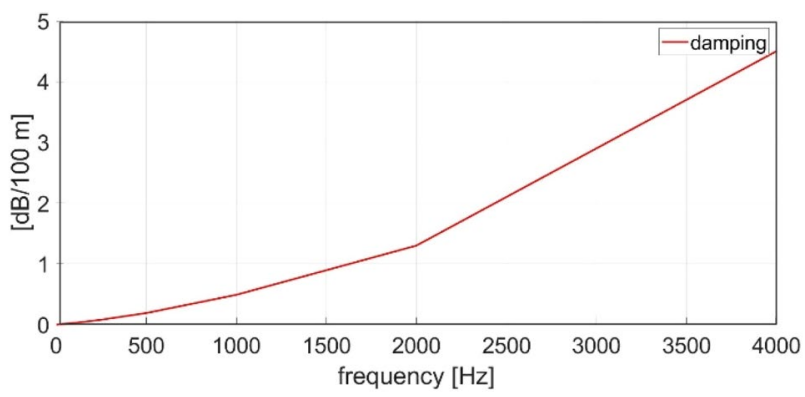

Fig. 16 Atmospheric damping per $100 \mathrm{~m}$ run length at $11{ }^{\circ} \mathrm{C}$ and $45 \%$ rel. humidity

\section{Optimization}

The existing dependency between distance and polar emission angle in the flight noise measurement data leads to the question, in how far optimal microphone positions and flight trajectories can be obtained in future flight noise measurements.

In the optimization study, the maximum absolute correlation coefficient $\operatorname{Corr}\left(X_{i}, X_{j}\right)$ between the quantities electric power consumption, emission angle, distance and bank angle is minimized. The minimization of correlations is interpreted as maximization of the statistical independence of the resulting measurement data. Thus, minimization of the correlations provides the mathematical requirements for an exact parameter estimation of the MLE (chapter 2.2) and can be understood as a maximization of the possible model quality. The bank angle is currently not part of the noise model but must be included in future modelings because of its meaning in turning flight. Thus, it is included in the optimization.

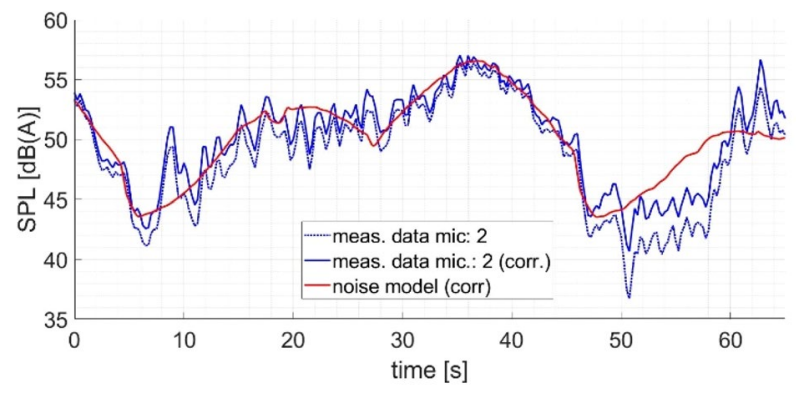

Fig. 17 SPL curve of corrected and not corrected measurement data and noise model (from corrected data)

The microphone displacements yield six optimization variables (OV). Each microphone can be displaced in two spatial directions until a maximum value in either positive or negative direction. The overall trajectory consists of three repetitions of a horizontal flight pattern, the altitudes of these three trajectory segments correspond to three more OV. The optimization is conducted using a genetic algorithm.

The base solution corresponds to an altitude of $60 \mathrm{~m}$ and a microphone positioning that is equivalent to the one that was chosen for the Manta Ray measurement during this work project.

The maximum correlation coefficient is 0.89 which agrees with the value of the horizontal flight model presented in chapter 4.1 .

Fig. 18 displays the optimization results in dependence of the maximum possible microphone displacement. During the first study, the altitudes are fixed to a value of $60 \mathrm{~m}$ and during the following two studies altitudes of $50-150 \mathrm{~m}$, respectively, 35-200 $\mathrm{m}$ are allowed. In each optimization result, at least one microphone is placed at the optimization variable border and the three flight altitudes are always distributed to the maximum and minimum possible values. The target function value decreases with increasing microphone displacement as well as with increasing spread of the altitudes.

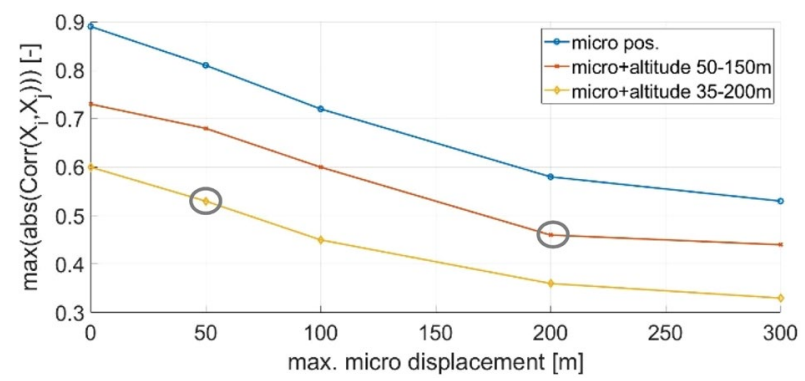

Fig. 18 Result of the optimization study 


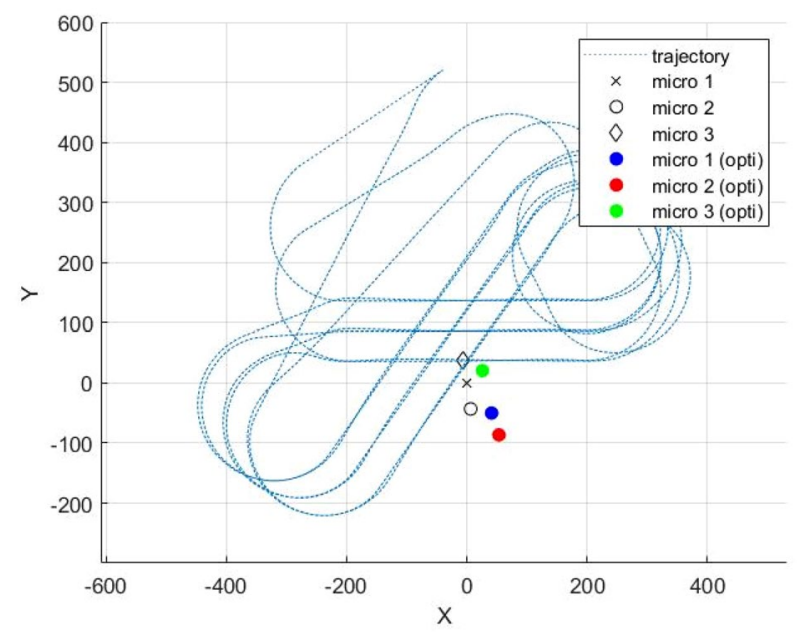

Fig. 19 Maximum microphone displacement $50 \mathrm{~m}$, altitude 35-200 m

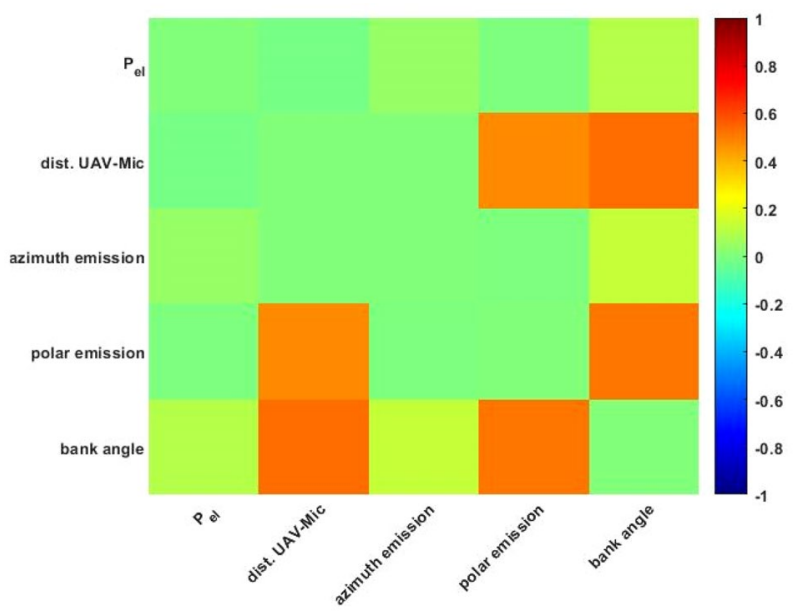

Fig. 20 Maximum microphone displacement $50 \mathrm{~m}$, altitude 35-200 m

A comparison of the optimization results marked in Fig. 18 shows that the correlation between bank angle and distance decreases with increasing microphone displacement as the microphones are not located in the center of the overall trajectory anymore (Fig. 19, 20, 21, 22).

These results point out that in future flight noise modelings high statistical independence of the measurement data should mainly be reached through high spreads of altitudes. High microphone displacements are to be looked at critically. On the one hand, high displacements lead to disadvantages in handling during measurement campaigns. On the other hand, the signal to noise ratio limits the maximum distance between UAV and microphones depending on the sound pressure levels of the UAV specific noise and the background noise. For this reason, measuring arrangements for future modelings should be optimized considering

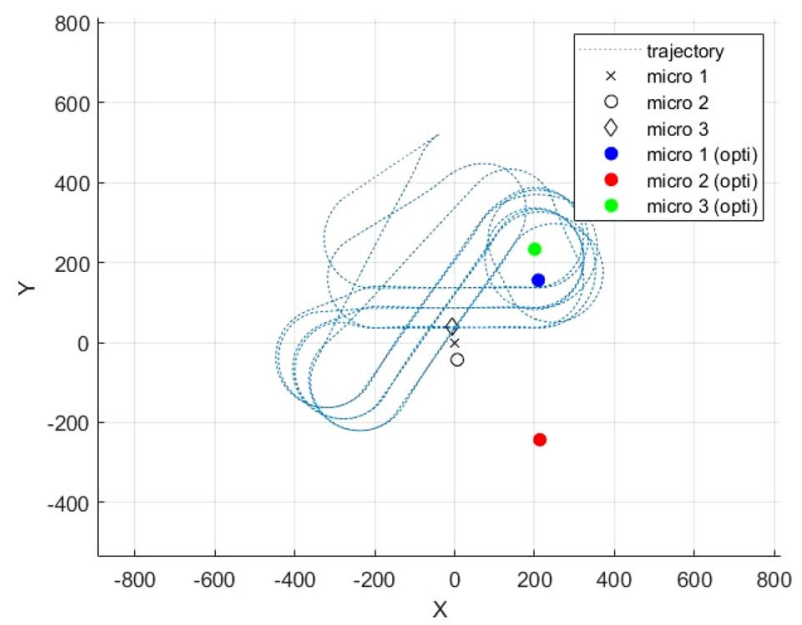

Fig. 21 Maximum microphone displacement $200 \mathrm{~m}$, altitude $50-150 \mathrm{~m}$

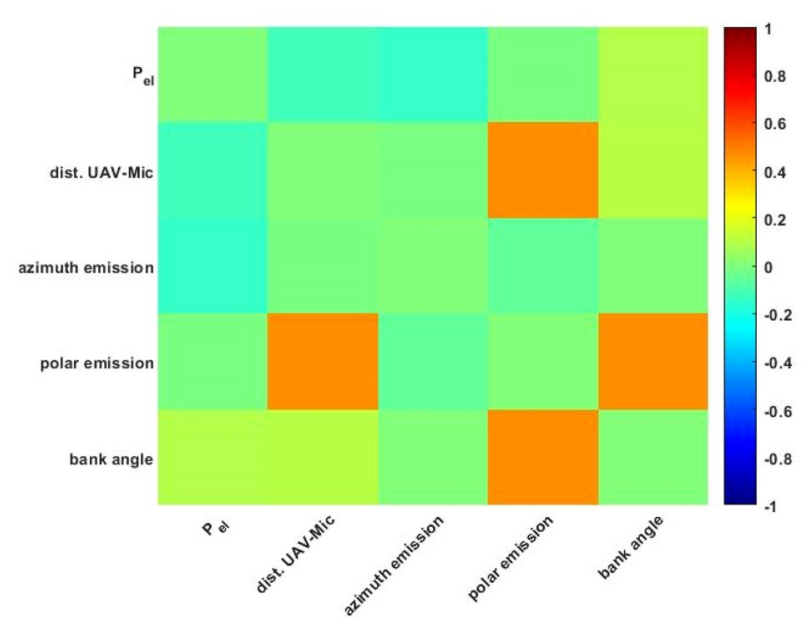

Fig. 22 Maximum microphone displacement $200 \mathrm{~m}$, altitude $50-150 \mathrm{~m}$

a distance restriction which accounts for the signal to noise ratio and practical aspects of microphone positioning.

\section{Summary}

Semi-empiric noise models were determined for horizontal flight as well as for climbing and decreasing vertical flight of an eVTOL UAV making use of a maximum likelihood estimator. As a compact analytical model approach was used, these models are suited for the integration into a geographical information system.

The horizontal flight model exhibits a significant directionality in noise emission and is capable of following the trend of the noise measurement data. A correlation analysis 


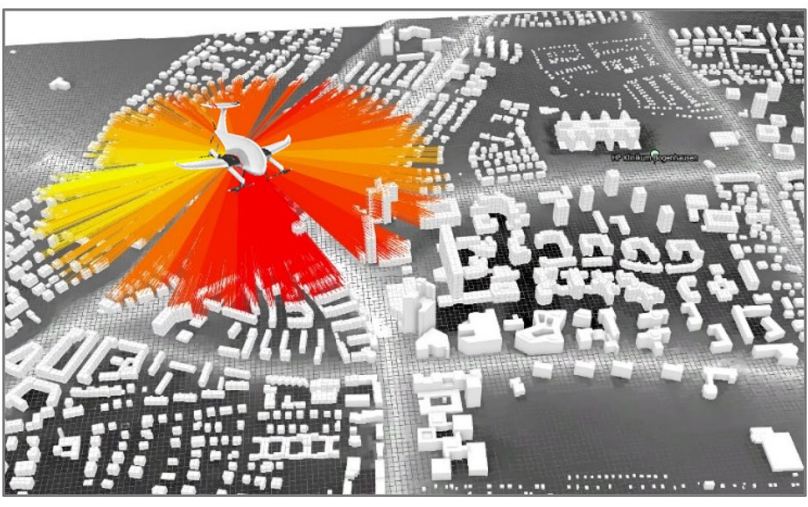

Fig. 23 Schematic representation of the Manta Ray horizontal flight noise model evaluated in a geo data model of an urban area, kindly provided by PSU and PW

shows that the deviations mainly occur in turning flight. Interaction noise can explain these deviations.

The vertical flight models show a less pronounced directionality in noise emission. They reproduce the measurement data trend in a lesser extent than the horizontal flight models. As a reason for this, the more complex physics of noise generation and propagation of four propellers is identified.

Statistical independence in the measurement data's control inputs are a precondition for the applicability of a maximum likelihood estimator. To provide this condition for future modelings, an optimization of microphone positions and altitudes of the single trajectory segments was conducted. The target was to minimize the maximum covariance in the control inputs. The results show that flying at different altitudes without changing the microphone positions reduces the maximum covariance already significantly.

\section{Outlook}

By achieving optimal microphone positions and flight trajectories regarding statistical independence of the noise measurement data, higher dimensional and, thus, more accurate flight modelings obtained by system identification are to be expected in future.

Furthermore, it is planned to conduct numerical aeroacoustic computations making use of a hybrid computational fluid dynamics (CFD)/Ffowcs-Williams-Hawkings(FW-H) approach. In this methodology, aeroacoustic sound sources are resolved by high-fidelity 3D Navier-Stokes CFD. A FW-H formulation of Lighthill's Acoustic Analogy [14] enables computation of the sound pressure in the acoustic far field. This approach promises to clarify the sound generation and propagation in vertical as well as hover flight which enables the development of suitable noise models.
Acknowledgements Within the project "Raumbezogene Modellierung zur Lärm-reduktion elektrischer Senkrechtstarter" (Spatial modeling for the noise reduction of electric vertical takeoff vehicles, acronym: RAUMOLES) a noise model of the cargo eVTOL UAV "Manta Ray" of project partner Phoenix-Wings $\mathrm{GmbH}$ was created by the Institute of Aircraft Design of the Technical University of Munich. One of the main targets was to integrate this noise model into a geographical information system (GIS) by the Prof. Schaller UmweltConsult GmbH and to demonstrate the noise impact of eVTOL operation in urban areas (see Fig. 23)

The authors express their gratitude to the Federal Ministry of Transport and Digital Infrastructure for sponsorship of the research work within the RAUMOLES project in the context of an mFund funding. They also thank their project partners from Prof. Schaller UmweltConsult $\mathrm{GmbH}$ and Phoenix-Wings GmbH for their continuous support.

Funding Open Access funding enabled and organized by Projekt DEAL. An mFund funding by the Federal Ministry of Transport and Digital Infrastructure enabled this work.

Open Access This article is licensed under a Creative Commons Attribution 4.0 International License, which permits use, sharing, adaptation, distribution and reproduction in any medium or format, as long as you give appropriate credit to the original author(s) and the source, provide a link to the Creative Commons licence, and indicate if changes were made. The images or other third party material in this article are included in the article's Creative Commons licence, unless indicated otherwise in a credit line to the material. If material is not included in the article's Creative Commons licence and your intended use is not permitted by statutory regulation or exceeds the permitted use, you will need to obtain permission directly from the copyright holder. To view a copy of this licence, visit http://creativecommons.org/licenses/by/4.0/.

\section{References}

1. Filippone, A., Bertsch, L.: Comparison of aircraft noise models with flyover data. J. Aircraft. Nr. 51(3), 1043-1046 (2014)

2. D. A. Conner und J. A. Page, 2002 A tool for low noise procedures design and community noise impact assessment: The Rotorcraft Noise Model (RNM).

3. Greenwood, E., Schmitz, F.H., Sickenberger, R.D.: Greenwood, Eric, Fredric H. Schmitz, and Richard D. Sickenberger. "A semiempirical noise modeling method for helicopter maneuvering flight operations. J Am Helicopter Soc 60(2), 1-13 (2015)

4. S. Speck, J. Wilberg und M. Hornung, ,An Approach for Aeroacoustic Footprint-Modeling of Low Altitude Platforms by Means of Time Domain System Identification," in 2013 Aviation Technology, Integration, and Operations Conference, Los Angeles, CA, 2013.

5. Phoenix-Wings GmbH.: https://phoenix-wings.de/ [Online]. Available: https://phoenix-wings.de/wp-content/uploads/2020/ 04/mr_port_bg.jpg. [Zugriff am 2508 2020]. Accessed 25 Aug 2020

6. Jategaonkar, R.: Flight Vehicle System Identification, Reston. AIAA, VA (2006)

7. Pott-Pollenske, M., Dobrzynski, W., Buchholz, H.: „Airframe Noise Characteristics from Flyover Measurements and Predictions, " in 12th AIAA/CEAS Aeroacoustics Conference. Massachusetts, Cambridge (2006)

8. Phoenix Wings GmbH.: https://phoenix-wings.de/ [Online]. Available: https://phoenix-wings.de/manta-ray/. [Zugriff am 27 08 2021]. Accessed 27 Aug 2021 
9. Marte, J.E., Kurtz, D.W.: A Review of Aerodynamic Noise From Propellers, Rotors, and Lift Fans. NASA, Washington, D.C. (1970)

10. T. Sinnige, K. Lynch, D. Ragni, G. Eitelberg und L. Veldhuis, „Aerodynamic and Aeroacoustc Effects of Pylon Training Edge Blowing on Pusher Propeller Installation, " in Proceedings of the 21st AIAA/CEAS aeroacoustics conference, Reston, 2015.

11. Yin, J., Stuermer, A., Aversano, M.: „Coupled uRANS and FW-H Analysis of Installed Pusher Propeller Aircraft Configurations, "in 15th AiAA/CEAS Aeroacoustics Conference. Florida, Miami (2009)

12. Botre M, Brentner KS, Horn JF, Wachspress D.: Validation of helicopter noise prediction system with flight data. In: Vertical Flight Society 75th Annual Forum \& Technology Display, Vol. 13. Philadelphia, PA (2019)

13. Müller, G., Möser, M.: Taschenbuch der Technischen Akustik. Springer, Berlin (2012)
14. Farassat, F.: Derivation of Formulations 1 and 1A of Farassat. Langley Research Center, Hampton, Virginia (2007)

15. Schwarzbach, M.: Raumbezogene Modellierung zur Lärmreduktion elektrischer Senkrechtstater; Acronym: RauMoLeS : Sachbericht/Schlussbericht zum F\&E Verbundvorhaben : Vorhabensbeschreibung des Partners Phoenix-Wings GmbH : Vorhabenslaufzeit: 01.06.2019-31.12.2019. Phoenix-Wings $\mathrm{GmbH}$, München (2020)

Publisher's Note Springer Nature remains neutral with regard to jurisdictional claims in published maps and institutional affiliations. 